\title{
Cáncer de vesícula biliar ¿La contaminación por bilis afecta el pronóstico?
}

\author{
Xabier de Aretxabala U. ${ }^{1,2}$, David Daroch M. ${ }^{1}$, Marcelo Vivanco L. ${ }^{1}$, Juan Hepp K. ${ }^{1}$, \\ Nicolás Solano V. ${ }^{3}$, Oriana Valenzuela C. ${ }^{3}$, Guillermo Rencoret P. ${ }^{1}$ e Iván Roa E. ${ }^{4}$
}

\section{Gallbladder Cancer: Does bile contamination affect the prognosis?}

Background: Incidental gallbladder cancer is observed in up to $3.5 \%$ of patients undergoing laparoscopic cholecystectomy. To study the role of wall perforation on the prognosis, we evaluated a series of patients in whom perforation occurred during the cholecystectomy. Materials and Method: 109 patients who underwent a laparoscopic cholecystectomy in whom final diagnosis was gallbladder cancer were the focus of the study. We divided the patients according the occurrence of spillage. Furthermore, patients with spillage were divided into two categories according the spillage magnitude. Results: Of the patients, spillage was documented in $32(29.3 \%)$. In 13 patients spillage was considered major. The median follow-up of patients was 36 months, while 35 (32.1\%) patients died during the follow-up. Five-year survival of all patients with spillage was not statistically different from the group without spillage. However, the group with major spillage had a statistically worse survival than the rest. A Cox regression analysis including age, gender, level of invasion and spillage category showed that major spillage was independently associated with a worse prognosis.

Key words: gallblader; cáncer; prognosis; perforation; cholecystectomy.

\section{Resumen}

Introducción: En algunas áreas el cáncer de la vesícula biliar se detecta en hasta el 3,5\% de los pacientes intervenidos por colelitiasis. Con el objetivo de evaluar el rol de la ruptura de la vesícula y la consiguiente contaminación por bilis, se evaluó una serie de pacientes portadores de cáncer de vesícula diagnosticado posterior a la colecistectomía. Materiales y Método: El estudio se efectuó en 109 pacientes en quienes se diagnosticó un cáncer de vesícula posterior a la colecistectomía. El grupo a estudiar se dividió de acuerdo a la ocurrencia o no de contaminación por bilis al momento de la colecistectomía, como también de acuerdo a la magnitud de ésta. Resultados: De los pacientes estudiados, en 32 se documentó la ocurrencia de contaminación por bilis al momento de la colecistectomía. De estos, en 13 la contaminación fue considerada mayor. El tiempo promedio de seguimiento fue de 33 meses, 35 pacientes $(32,1 \%)$ fallecieron durante el seguimiento. La sobrevida media de la totalidad de la serie que tuvo contaminación por bilis no se diferenció de los pacientes sin contaminación. Sin embargo, el grupo que tuvo una contaminación catalogada como mayor, presentó una sobrevida estadísticamente inferior al resto de los pacientes. Finalmente, se realizó un análisis mediante el modelo de regresión de COX que incluyó edad, género, nivel de invasión y tipo de contaminación, resultando la existencia de contaminación mayor por bilis un factor independientemente asociado al pronóstico. Conclusión: La presencia de ruptura vesicular y contaminación mayor por bilis debiera considerarse un factor pronóstico.

Palabras clave: vesícula; cáncer; pronóstico; perforación; colecistectomía.

\section{Introducción}

El cáncer de la vesícula biliar se caracteriza por ser una enfermedad de baja frecuencia, generalmente, asociada a un mal pronóstico, especialmente cuando es detectada en estadios avanzados.
Los factores pronósticos más importantes son: la profundidad de invasión en la pared vesicular, la invasión de órganos vecinos y el compromiso linfático ${ }^{1-5}$.

Desde el comienzo del empleo de la colecistectomía laparoscópica, el número de tumores incidenta-
'Departamento de Cirugía Clínica Alemana. Santiago, Chile.

2Departamento de Cirugía Hospital Fuerza Aérea. Santiago, Chile.

${ }^{3}$ Departamento de Cirugía Hospital Quillota. Quillota Chile. ${ }^{4}$ Creative Bioscience. Santiago Chile.

Recibido el 10 de junio de 2019 y aceptado para publicación el 19 de agosto de 2019.

Correspondencia a: Dr. Xabier de Aretxabala U. xdearetxabala@alemana.cl 
les ha aumentado, siendo estos los asociados a una mayor sobrevida ${ }^{2,3,5}$.

Por otra parte, hay estudios que han mostrado la existencia de una relación entre la perforación de la vesícula y una mayor recurrencia, principalmente, cuando el diagnóstico tumoral es posterior a la colecistectomía $^{6-9}$. A pesar de lo anterior, no existe consenso en catalogar como un factor de riesgo a la ruptura de la vesícula, ni tampoco en proponer acciones terapéuticas. Considerando la importancia de esta relación, estudiamos el efecto que tendría la contaminación peritoneal por bilis durante la colecistectomía, con la sobrevida de los portadores de un cáncer de vesícula biliar diagnosticado posterior al estudio de la pieza de colecistectomía.

\section{Materiales y Método}

Desde el año 1988, el autor lleva un registro prospectivo de pacientes portadores de un cáncer de vesícula potencialmente resecables que le son derivados para su manejo. El estudio fue definido como una cohorte histórica. El término potencialmente resecable dice relación a un tumor diagnosticado durante la colecistectomía y que fue resecado completamente o es potencialmente resecable en una reintervención. Para evaluar la presencia de contaminación peritoneal por bilis proveniente de una ruptura de la vesícula, nos basamos en la descripción del protocolo operatorio y consultando a los cirujanos que efectuaron la colecistectomía.

El grado de contaminación por bilis fue dividido en mayor, si había presencia de cálculos en el peritoneo o necrosis vesicular, y en menor, si no había presencia de estos factores.

La etapificación del cáncer fue definida de acuerdo a la octava edición del Manual de la American Joint Comitte on Cancer ${ }^{10}$.

En relación al manejo, los pacientes con tumores in situ o mucosos fueron considerados tratados con la colecistectomía. Los pacientes portadores de una enfermedad más extensa fueron etapificados empleando tomografía axial computada y considerados para una reexploración con la finalidad de efectuar la resección del lecho de la vesícula biliar y la linfadenectomía del pedículo hepático.

Contraindicación para efectuar la reexploración fue la presencia de tumor fuera de las áreas a resecar en una colecistectomía extendida formal.

El tipo de abordaje dependió del periodo de tiempo en que estas realizaron. Al comienzo del periodo en estudio los pacientes se sometieron a una clásica reintervención abierta mientras que durante los últimos 8 años, la reintervención fue laparoscópica.

Para el análisis estadístico, se empleó el programa SPSS versión 240 Las comparaciones univariables fueron efectuadas mediante el test de $\chi^{2}$, para variables dicotómicas mientras que para variables numéricas paramétricas se empleó el test de Student. Para variables no paramétricas, se empleó el test de Mann-Whitney.

La sobrevida se calculó usando el método de Kaplan-Meier y las curvas se compararon mediante el test de log Rank. Las diferencias se consideraron estadísticamente significativas cuando $\mathrm{p}<=0,05$. Un modelo de regresión de COX fue empleado para determinar la existencia de variables independientes en relación a la sobrevida.

\section{Resultados}

Entre los años 1988 y 2016, 442 pacientes portadores de un cáncer de vesícula biliar, componen la base de datos del autor, de éstos 109 fueron inicialmente intervenidos mediante una colecistectomía laparoscópica y son la base del estudio. Diecinueve pacientes fueron de sexo masculino y 90 femenino. La edad media de la serie fue de 58 años.

Nueve pacientes debieron convertirse a una colecistectomía abierta debido a dificultades técnicas durante la laparoscopía.

Contaminación por bilis, se documentó en 32 pacientes (29\%). De estos, 13 tuvieron un derrame de bilis catalogado como mayor. En 8 de estos pacientes, la caída de cálculos fue documentada en el protocolo operatorio, mientras que en 3 pacientes, en quienes no se había documentado la caída de cálculos en el protocolo operatorio, se encontraron cálculos al momento de la reintervención. Otros 2 pacientes en quienes se documentó la existencia de extensa necrosis de la pared de la vesícula y derrame de bilis, fueron también catalogados como contaminación mayor.

En las Tablas 1 y 2 se observa la relación entre la contaminación por bilis y variables tales como edad, género e invasión de la pared, no observándose asociaciones estadísticamente significativas.

El diagnóstico de cáncer se sospechó en 9 pacientes, previo a la colecistectomía, en 16 al momento de ésta y en 84 no se sospechó. De los 9 pacientes en quienes el diagnóstico fue sospechado antes de la colecistectomía, ninguno presentó contaminación por bilis. Sin embargo, entre los 16 pacientes en que se sospechó el diagnóstico de tumor vesicular durante la cirugía, dos tuvieron contaminación de la cavidad peritoneal por bilis. 
Tabla 1. Distribución en relación a la ocurrencia de contaminación con bilis

\begin{tabular}{|lccc|}
\hline Contaminación & No & Sí & p \\
\hline Edad (media) & $59($ IC $95 \%$ 57-61) & $56($ IC 95\% 53-60) & 0,591 \\
Género M/F & $62 / 15$ & $28 / 4$ & 0,382 \\
Invasión & & & 0,574 \\
$\quad$ In situ/mucosa & $3(3,9 \%)$ & $4(12,5 \%)$ & \\
Muscular & $15(19,4 \%)$ & $5(15,6 \%)$ & \\
Subserosa & $41(53,2 \%)$ & $16(50 \%)$ & 0,018 \\
Serosa & $16(20,7 \%)$ & $6(18,7 \%)$ & $(3,1 \%)$ \\
Adiposo & $2(2,5 \%)$ & $21($ rango 3-180) & \\
Sobrevida (media) & $55($ rango 2-240) & \\
\hline
\end{tabular}

Tabla 2. Distribución en relación a los pacientes que sufrieron contaminación mayor

\begin{tabular}{|lccc|}
\hline Contaminación mayor & No & Sí & p \\
\hline Edad (media) & $58($ IC $95 \%$ 56-61) & $57($ IC 95\% 52-62) & 0,294 \\
Género M/F & $78 / 18$ & $12 / 1$ & 0,324 \\
Invasión & $5(\%)$ & & 0,519 \\
In situ/mucosa & $19(\%)$ & $2(\%)$ & \\
Muscular & $50(\%)$ & $1 \%)$ & \\
Subseroso & $19(\%)$ & $3(\%)$ & 0,015 \\
Serosa & $3(\%)$ & $0(\%)$ & \\
Adiposo & 43 (range 2-240) & 12 (range 4-84) \\
Sobrevida (media) & & \\
\hline
\end{tabular}

En 31 pacientes, la colecistectomía fue el único procedimiento efectuado. La existencia de compromiso tumoral limitado a la mucosa y el rechazo del paciente a someterse a un procedimiento adicional fueron las causas para no reoperar a los pacientes. Además, en 4 pacientes durante la etapificación, se observó diseminación tumoral fuera de las áreas a resecar, siendo esto indicación de no reintervenir.

Finalmente 78 pacientes fueron reoperados. De estos pacientes, 3 no fueron resecados debido a malas condiciones médicas, mientras que 23 tuvieron extensión del tumor mas allá de las áreas a resecar siendo declarados irresecables.

La distribución de los pacientes según el procedimiento efectuado y si hubo contaminación por bilis se muestra en las Tablas 3 y 4, sin observarse diferencias estadísticamente significativas entre los grupos.

El tiempo de seguimiento medio posterior a la colecistectomía fue de 36 meses (rango 2 a 240). Al momento de este reporte, $35(32 \%)$ pacientes han muerto debido al cáncer vesicular. La sobrevida media del grupo de pacientes que tuvieron conta- minación por bilis fue de 21 meses (rango 3-180), en cambio, en el grupo sin contaminación fue de 55 meses (rango 2-240) $(\mathrm{p}=0,018)$. La sobrevida media del grupo que tuvo contaminación mayor fue de 12 meses (rango 4-84), mientras que, el grupo sin contaminación mayor fue de 43 meses (rango 2-240) $(\mathrm{p}=0,015)$ (Tablas 1 y 2$)$.

Las Figuras 1 y 2 muestran la sobrevida de los grupos de pacientes de acuerdo a la existencia de derrame biliar y al grado de esta. El grupo total de pacientes que tuvo contaminación por bilis, independiente de su magnitud, no mostró diferencias significativas en la sobrevida comparado con el grupo de pacientes sin contaminación (log-rank 0,455). Sin embargo, los pacientes que tuvieron contaminación mayor de bilis tuvieron una diferencia significativa en la sobrevida al compararlo con el resto de los pacientes.

Finalmente, se realizó un modelo de regresión de Cox que incluyó la edad, género, profundidad de invasión en la pared y presencia de contaminación. En este modelo solo la contaminación mayor se observó asociarse a una peor sobrevida $(p=0,016)$ (Tabla 5). 


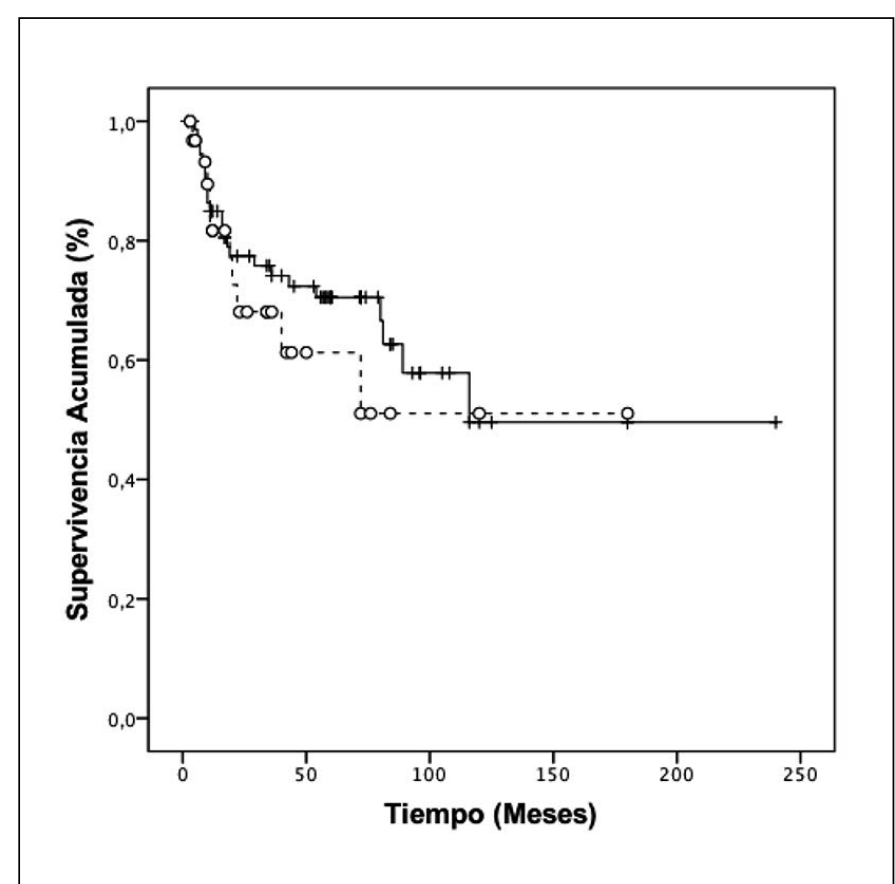

Figura 1. Curvas de sobrevida en pacientes que presentaron contaminación versus los que no presentaron. Línea continua: Pacientes sin contaminación. Línea punteada: Pacientes con contaminación.

Tabla 3. Procedimientos realizados en relación a la ocurrencia de contaminación

\begin{tabular}{|lccc|}
\hline Contaminación & No & Sí & p \\
\hline Tipo de procedimiento & & & 0,884 \\
\hline Solo colecistectomía & 21 & 10 & \\
\hline Resección & 39 & 16 & \\
\hline Laparotomía exploradora & 17 & 6 & \\
\hline Total & 77 & 32 \\
\hline
\end{tabular}

Tabla 5. Modelo de regresión de COX

\begin{tabular}{|lccc|}
\hline Variable & p & $\begin{array}{c}\text { Hazard } \\
\text { Ratio }\end{array}$ & IC 95\% \\
\hline Edad & 0,688 & - & - \\
Sexo & 0,844 & - & - \\
$\begin{array}{l}\text { Perforación } \\
\text { mayor }\end{array}$ & 0,016 & 0,314 & $0,122-0,809$ \\
Invasión & 0,575 & - & - \\
\hline
\end{tabular}

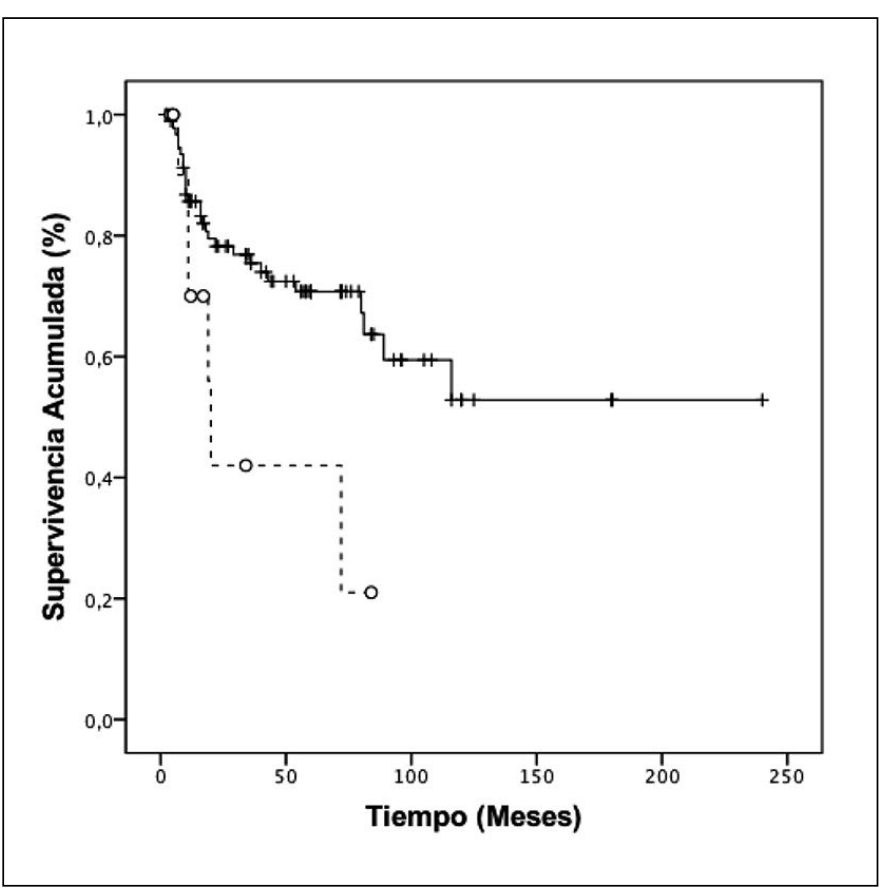

Figura 2. Curvas de sobrevida de los pacientes que presentaron contaminación mayor versus el resto de pacientes. Línea continua: Pacientes sin contaminación y contaminación menor. Línea punteada: Pacientes con contaminación mayor.

Tabla 4. Grado de contaminación en relación al procedimiento efectuado

\begin{tabular}{|lccc|}
\hline Contaminación mayor & No & Sí & p \\
\hline Tipo de procedimiento & & & 0,112 \\
\hline Solo colecistectomía & 30 & 1 & \\
\hline Resección & 48 & 7 & \\
\hline Laparotomía exploradora & 18 & 5 & \\
\hline Total & 96 & 13 & \\
\hline
\end{tabular}

\section{Discusión}

Desde el inicio de la colecistectomía laparoscópica, se han publicado estudios que asocian este procedimiento con una peor sobrevida ${ }^{5-9}$. La contaminación peritoneal por bilis $\mathrm{y}$ células neoplásicas debido a la ruptura de la vesícula, sería la explicación para este problema. Sin embargo, estudio clínicos de neoplasias malignas gastrointestinales muestran que la laparoscopia no se ha asociado a una mayor recurrencia, comparado con cirugía abierta ${ }^{11-16}$. 
La mayoría de los pacientes portadores de un cáncer de vesícula asociados a un mejor pronóstico, son aquellos detectados posterior a la colecistectomía, en el examen de la pieza de colecistectomía. Es justamente en este grupo de pacientes donde el conocimiento del efecto de la contaminación peritoneal por bilis debido a la perforación involuntaria de la vesícula cobra mayor importancia.

Del análisis de la literatura publicada, observamos que pocos autores han estudiado este hecho. Goetze reporta los resultados obtenidos del análisis del registro sobre cáncer incidental de la Sociedad Alemana de Cirugía mostrando que la ruptura vesicular se asocia a la presencia de una mayor recurrencia $^{6}$. Lee publicó su experiencia con una serie de 28 pacientes portadores de un cáncer vesicular sometidos a una colecistectomía laparoscópica en quienes el tumor se detectó posterior a la colecistectomía ${ }^{7}$. En esta serie, en $12(43 \%)$ pacientes se documentó contaminación por bilis al momento de la cirugía, resultando en un peor pronóstico comparado a aquellos en que no ocurrió contaminación. Sin embargo, en este grupo, la proporción de pacientes con resecciones no curativas y estadios avanzados fue alta, lo que asociado al bajo número de pacientes estudiados le resta validez a las conclusiones. Ahmad, enfatizó esta posible relación describiendo un paciente que desarrolló un tumor posterior a la colecistectomía, observando cálculos biliares incrustados en el tumor ${ }^{17}$.

El presente estudio otorga información adicional relacionada a la posible relación entre colecistectomía laparoscópica y sobrevida en cáncer de vesícula biliar. Lo primero a destacar es la alta frecuencia de contaminación por bilis en nuestra serie. El 29\% de los pacientes sometidos a una colecistectomía laparoscópica en esta serie, tuvieron una perforación de la pared con la consiguiente contaminación por bilis. Esta cifra es coincidente con otras publicaciones que han descrito frecuencias de perforación incidental de la vesícula que van de un $13 \%$ a un $40 \%{ }^{5}$. Las variaciones en las cifras anteriores pueden ser explicadas no solo por diferencias técnicas, sino también por casos que no son reportados. En nuestra serie, la cifra de contaminación por bilis se obtuvo no solo del análisis del protocolo operatorio, sino también de la entrevista con el cirujano que realizó la colecistectomía y de los hallazgos de las reintervenciones. De hecho, en 3 pacientes en quienes no se había reportado contaminación, se encontraron cálculos libres en la cavidad abdominal al momento de la reoperación. La importancia de lo anterior radica en la necesidad del empleo de una técnica cuidadosa al momento de realizar una colecistectomía y convertir a cirugía abierta en caso de sospecha de cáncer vesicular, por el riesgo de diseminar el tumor. Desafortunadamente, del análisis de nuestra serie, se observa que esta regla no es seguida de manera uniforme incluso en un país con una alta incidencia de cáncer de vesícula como Chile, ya que en los pacientes en quienes se sospechó la existencia de un tumor previo al estudio histológico no se modificó la conducta.

Este estudio sugiere la importancia de la magnitud de la contaminación peritoneal por bilis en el pronóstico de la enfermedad, más que su sola existencia. Por un lado, en los casos en que la contaminación por bilis fue definida como menor, no se alteró la sobrevida, lo cual no ocurrió en los casos en los que la contaminación fue catalogada como mayor, donde sí se evidencia una disminución en la sobrevida.

Aunque una de las principales limitaciones de este estudio es su carácter retrospectivo y el relativo bajo número de pacientes en cada grupo estudiado, debido a la baja frecuencia de la enfermedad, esta serie es una de las más grandes publicadas que estudien esta relación.

De lo anterior, podríamos concluir que la contaminación por bilis en pacientes con cáncer de vesícula parece tener un rol en el pronóstico, en quienes el diagnóstico se realiza posterior a la colecistectomía. La confirmación de este hecho determina que la contaminación por bilis es un factor pronóstico y es necesario el empleo de nuevas alternativas terapéuticas frente a la ocurrencia de esta complicación.

\section{Responsabilidades éticas}

Protección de personas y animales. Los autores declaran que para esta investigación no se han realizado experimentos en seres humanos ni en animales.

Confidencialidad de los datos. Los autores declaran que en este artículo no aparecen datos de pacientes.

Conflictos de interés: no hay. 


\section{Bibliografía}

1. Stinton LM, Shaffer EA. Epidemiology of gallbladder disease: cholelithiasis and cancer. Gut and Liver 2012;6: $172-87$.

2. de Aretxabala X, Roa I, Burgos L, Losada H, Roa JC, Mora J, et al. Gallbladder cancer: an analysis of a series of 139 patients with invasion restricted to the subserosal layer. $\mathrm{J}$ Gastrointest Surg. 2006;10:186-92.

3. Wistuba II, Gaazdar AF. Gallbladder cancer: lessons from a rare tumor. Nat Rev Cancer 2004;4:695-706.

4. Tantia O, Jain M, Khannas S, Sen B. Incidental carcinoma of the gallbladder during laparoscopic cholecystectomy for symptomatic gallstone disease. Surg Endosc. 2009;23:2021-6.

5. Jorcidek K, Guest R, Harrison CM, Kendall TJ, Garden O J, Wigmore SJ. Systematic review of management of incidental gallbladder cáncer after cholecystectomy. Br J Surg. 2019;106:3245.

6. Goetze TO, Paoluci V. Use of retrieval bags in incidental gallbladder cancer World J Surg. 2009;33:2161-5.
7. Lee JM, Kim BW, Kim WH, Wang HJ, Kim MW. Clinical implications of bile spillage in patients undergoing cholecystectomy for gallbladder cancer. Am Surg. 2011;7:697-701.

8. Ouchi K, Mikuni J, Kakugawa Y. The 30th Annual Congress of the Japanese Society of Biliary Surgery. Laparoscopic cholecystectomy for gallbladder carcinoma: results of a Japanese survey of 498 patients. J Hepatobiliary Pancreat Surg. 2002;9:256-60.

9. Z' graggen K, Birrer S, Maurer CA, Wehrli H, Klaiber C, Baer HU. Incidence of port site recurrence after laparoscopic cholecystectomy for preoperatively unsuspected gallbladder carcinoma. Surgery 1998;124:831-8.

10. Chun YS, Pawlik TM, Vauthey JN. 8th edition of the AJCC cancer and staging Manual: Pancreas and Hepatobiliary Cancers. Ann Surg Oncol. 2018;25:845-7.

11. Maxwell-Armstrong CA, Robinson $\mathrm{M} \mathrm{H}$, Scholefield JH. Laparoscopic colo rectal cáncer surgery. Am J Surg. 2000;179:5007.

12. Deijen CL, Vasmel JE, Lange-deKlerk ES, Cuesta MA, Coene PLO,Lange JF et al. Ten year outcomes of randomised trial of laparoscopic versus open surgery for colon cancer. Surg Endosc. 2017;31:260715.

13. Honda M, Hiki N, Kinoshita T, Yabusaki $\mathrm{H}$, Abe $\mathrm{T}$, Nunobe $\mathrm{S}$, et al. Long term outcomes of laparoscopic versus open surgery for clinical stage I gastric cancer: The LOC-1 Study. Ann Surg. 2016;264:214-22.

14. Jiang JB, Jiang K, Dai Y, Wang RX, Wu WZ, Wang JJ, et al. Laparoscopic versus open surgery for mid-low rectal cancer: A systematic review and meta-analysys on short and long term outcomes.

J Gastrointest Surgery 2015;19: 1497-512.

15. Lee JJ, Conneely JB, Smoot RL, Gallinger S, Greig PD, Moulton CA, et al. Laparoscopic versus open liver resection for hepatocellular carcinoma at a North American Centre: a 2-to-1 matched pair analysis. HPB 2015;17:304-10.

16. Ugarte F. Laparoscopic cholecystectomy port seeding from a colon carcinoma Am Surg. 1995;820-1.

17. Ahmad J, Mayne AI, Zen Y, Loughrey MB, Kelly P, Taylor M. Spilled gallstones during laparoscopic cholecystectomy. Ann R Coll Surg Engl. 2014;96:18-20. 